\title{
Percutaneous left atrial appendage occlusion for stroke prevention in patients with atrial fibrillation
}

\author{
Hans-Christoph Diener*,1(i) \& Ulf Landmesser ${ }^{2}$ \\ ${ }^{1}$ Institute for Medical Informatics, Biometry \& Epidemiology, Medical Faculty, University Duisburg-Essen, Essen, Germany \\ ${ }^{2}$ Department of Cardiology, Campus Benjamin Franklin (CBF), Charité - Universitätsmedizin Berlin, Berlin, Germany; Berlin Institute \\ of Health (BIH), German Center of Cardiovascular Research (DZHK), Partner Site Berlin, Berlin, Germany \\ *Author for correspondence: hans.diener@uk-essen.de
}

Patients with atrial fibrillation (AF) have a fivefold higher risk of stroke than persons in sinus rhythm. Effective stroke prevention is achieved with oral anticoagulants such as vitamin $\mathrm{K}$ antagonists or nonvitamin $\mathrm{K}$ oral anticoagulants. An alternative for stroke prevention in patients with AF is the closure of the left atrial appendage (LAA) with a percutaneously applied closure system. The two large randomized studies PROTECT-AF and PREVAIL failed to show superiority of LAA closure over anticoagulation in patients with AF. Meta-analyses of studies and registries, however, suggest that LAA closure has particular advantages with regard to the reduction of severe bleeding complications. Currently, several prospective randomized studies are being conducted in different patient populations to evaluate the benefit of LAA closure in comparison to standard of care. Currently, LAA closure is recommended in patients after intracranial hemorrhage, with advanced renal failure, after severe gastrointestinal bleeding, in patients with a high risk of recurrent ischemic stroke and elderly patients with high risk of bleeding and falling.

First draft submitted: 15 January 2020; Accepted for publication: 14 April 2020; Published online: 3 July 2020

Keywords: antithrombotic therapy $\bullet$ atrial fibrillation $\bullet$ bleeding complications $\bullet$ left atrial appendage occlusion $\bullet$ stroke

Atrial fibrillation (AF) is an important risk factor for ischemic strokes. The risk of stroke is five-times higher in patients with AF compared with patients in sinus rhythm. Stroke prevention in patients with AF is achieved by oral anticoagulation with either vitamin $\mathrm{K}$ antagonists or nonvitamin $\mathrm{K}$ oral anticoagulants (NOACs) [1]. Compared with vitamin $\mathrm{K}$ antagonists, NOACs as a group are more effective in preventing strokes and have a significantly better safety profile with regards to intracranial bleeding [2]. In patients with AF, vitamin $\mathrm{K}$ antagonists, compared with placebo, achieve a $68 \%$ risk reduction for strokes [3]. This risk reduction can be further increased by $10-15 \%$ by using NOACs. Alternative methods for stroke prevention in AF would therefore have to achieve an approximately $80 \%$ risk reduction, similar to the use of NOACs compared with no therapy or placebo. As a possible alternative, interventional methods such as the closure of the left atrial appendage (LAA) with percutaneous catheter-based placement of devices such as the Amulet (Abbott, IL, USA) or WATCHMAN (Boston Scientific, MA, USA) LAA occluders have been developed for stroke prevention in patients with AF [4]. There are two important end points in trials comparing LAA occlusion with oral anticoagulation: the reduction in the rate of ischemic stroke and systemic embolism (SE) and to reduce the risk of major and fatal bleeding. Another alternative is the surgical closure of the LAA during planned cardiac surgery.

LAA closure was originally developed as an alternative to oral anticoagulation with vitamin $\mathrm{K}$ antagonists. As expected, there was no superiority of LAA closure for stroke prevention compared with warfarin (see below). After the approval of the first closure systems, the indication for LAA closure was changed and most procedures are now performed in patients with contraindications for long-term oral anticoagulation.

Future Medicine 
Table 1. Randomized trials comparing left atrial appendage occlusion with antithrombotic therapy.

\begin{tabular}{|c|c|c|c|c|c|c|c|}
\hline \multirow[t]{2}{*}{ Study } & \multirow[t]{2}{*}{ Device } & \multirow[t]{2}{*}{ Control } & \multicolumn{2}{|c|}{ Primary outcome ${ }^{\dagger}$} & \multicolumn{2}{|c|}{ Ischemic stroke } & \multirow[t]{2}{*}{ Ref. } \\
\hline & & & Device & Control & Device & Control & \\
\hline PREVAIL & 269 & 138 & $6.4 \%$ & $6.3 \%$ & $1.9 \%$ & $0.7 \%$ & [6] \\
\hline PROTECT AF & 463 & 244 & $2.3 \%$ & $3.8 \%$ & $1.4 \%$ & $1.1 \%$ & [5] \\
\hline PRAGUE 17 & 213 & 202 & $11 \%$ & $13 \%$ & NA & NA & [7] \\
\hline
\end{tabular}

†PREVAIL: Stroke, systemic embolism (SE), cardiovascular death.

PROTECT: Stroke, SE, cardiovascular death. PRAGUE-17: Cardiovascular death, stroke, transient ischemic attack, SE, significant bleeding, complications.

NA: Data not available.

\section{Randomized studies}

The first randomized study comparing the closure of the LAA with oral anticoagulation with a vitamin K antagonist was the PROTECT-AF study [5]. It was a multicenter randomized open-label study at 59 hospitals. The study included 707 patients with AF and at least one additional risk factor for stroke. The study enrolled patients between February 2005 and June 2008 and patients were followed up until 2012. In the study, 463 patients received LAA closure and 244 were anticoagulated with warfarin. The primary end point was the combination of stroke, SE and cardiovascular death (Table 1).

The patients were 72 years old on average and the mean CHADS2 score was 2.2. Two-thirds of patients had been in AF for more than 1 year. After a mean observation period of 3.8 years, 39 primary events occurred in the 463 patients in the LAA closure group corresponding to $8.4 \%$ and 34 events occurred in the 244 patients in the anticoagulation group corresponding to $13.9 \%$. This corresponds to a rate ratio of 0.60 with a $95 \%$ CI of $0.41-1.05$. Within the end points, stroke frequency was 1.5 versus $2.2 \%$ and cardiovascular death 1.0 versus $2.4 \%$. The incidence of serious adverse events was $3.6 \%$ in the device group compared with $3.1 \%$ in the warfarin group. The most common adverse events in the device group were pericardial tamponades and major bleeding in both groups. Pericardial effusions were observed in $4.8 \%$ of patients in the device group and severe bleeding complication in $4.8 \%$ of the device group and $7.4 \%$ in the warfarin group.

In summary, the PROTECT-AF study showed a strong trend in favor of LAA closure for the primary end point of stroke, $\mathrm{SE}$ and cardiovascular death. However, the significance level was narrowly missed.

The second study was the PREVAIL trial [6]. This randomized study investigated the efficacy and safety of the WATCHMAN closure system. Patients with AF and a CHADS2 score of $\geq 2$ were included. Patients were randomized in a 2:1 ratio of LAA occlusion or warfarin. The primary efficacy end point was the combination of stroke, SE and cardiovascular death. In the LAA group 269 patients were randomized, and similarly 138 patients were randomized in the control group. The mean age of patients was 74.5 years and $70 \%$ were male. After 18 months, the frequency of the primary end point was $6.4 \%$ in the device group and $6.3 \%$ in the control group. This corresponds to a rate ratio of 1.07 with a $95 \% \mathrm{CI}$ of $0.57-1.89$. Ischemic strokes occurred five-times in the device group and one stroke occurred in the control group. This corresponds to 1.9 versus $0.7 \%$. The frequency of pericardial effusions in the device group was $0.4 \%$. In summary, the PREVAIL study did not demonstrate superiority of LAA closure over anticoagulation with a vitamin $\mathrm{K}$ antagonist.

A meta-analysis analyzed the data of the PREVAIL and PROTECT-AF studies over a 5-year observation period [8]. The device group included 732 patients and the control group included 382 patients. The combined primary end point of stroke, SE and cardiovascular death did not differ between LAA closure and antithrombotic therapy. The hazard ratio (HR) was 0.820 . This was also true for strokes and SEs with a HR of 0.961 . The annual frequency of ischemic strokes was numerically higher for LAA closure compared with the control group with 1.6 versus $0.95 \%$. The HR was 1.71. Significant benefits of LAA closure were observed for hemorrhagic stroke at 0.17 versus $0.87 \%$ (HR: 0.20 ), stroke with severe disability at 0.44 versus $1.0 \%$ (HR: 0.45 ) and all-cause mortality at 3.6 versus $4.9 \%$ (HR: 0.73 ). There was no significant difference for severe bleeding. For severe nonprocedural related bleeding, the device group was superior with a rate of 1.7 versus $3.6 \%$ with a HR of 0.48 .

The 5-year outcomes of PROTECT-AF and PREVAIL were reported in a meta-analysis [8]. For the composite of stroke, SE and cardiac death the WATCHMAN device was noninferior to warfarin. Major bleeding, cerebral hemorrhage and overall mortality favored LAA occlusion.

Looking at these two studies from today's perspective, it is obvious that a number of shortcomings in the design of the studies needs to be considered when interpreting these results. Considering the frequency of the end points, 
the number of patients was too low and the observation period too short. The main criticism, however, is that these studies included patients who could be orally anticoagulated without restrictions.

The PRAGUE-17 study was a randomized trial in patients with AF who were randomized either to LAA occlusion or a NOAC [7]. There were 213 patients in the intervention group and 202 patients in the NOAC group. Postinterventionally, patients received dual antiplatelet therapy for 3 months. The mean observation period was 21 months, the mean age of the patients was 73 years. The primary end point of cardiovascular death, stroke, transient ischemic attack, SE and significant bleeding complication or percentage of complications did not differ between the two treatment groups. The HR was 0.84 . The study may have been too small to show a significant difference.

\section{Meta-analyses \& systematic reviews}

There are significantly more meta-analyses on stroke prevention in AF by occlusion of the left atrium than there are randomized studies. However, the meta-analyses usually also include data from open registries. The metaanalysis by Bode et al. included 16 studies with 1759 patients who received LAA closure [9]. The authors created a fictitious control group from data of the ATRIA study, the Danish national patient registry and the results of three studies comparing NOACs with warfarin. LAA closure reduced the risk of stroke compared with no therapy or acetylsalicylic acid with a relative risk of 0.34 (95\% CI: $0.25-0.46)$ and compared with warfarin with a relative risk of 0.65 (95\% CI: 0.46-0.91). Compared with $2 \times 150$-mg dabigatran or $2 \times 5$-mg apixaban, LAA closure had a higher risk of stroke with a relative risk of 1.69 and 1.59 , respectively. At that time, $6.1 \%$ of patients had a technical failure of LAA closure. Pericardial tamponade occurred in $2.4 \%$ of patients.

The meta-analysis by Li et al. investigated the 1-year efficacy and safety of LAA closure compared with NOACs for stroke prevention in patients with $\mathrm{AF}$ [10]. In this analysis six randomized studies and 27 observational studies were included, Superiority of LAAO over NOACs was notdemonstrated in terms of stroke prevention. LAAO was found to be associated with a lower rate of both thromboembolic and hemorrhagic events as compared with NOACs in observational studies.. A network meta-analysis of the randomized studies showed that the LAA closure was less effective than NOACs for stroke prevention with an odds ratio (OR) of 0.86 .

A further network meta-analysis was conducted by Sahay et al. [11]. This study concluded 19 randomized studies with 87,831 patients with AF. The indirect comparison with warfarin as the comparator showed a benefit of LAA closure compared with placebo for the end point stroke and SE with a HR of 0.24 (95\% CI: $0.11-0.52$ ) and treatment with antiplatelet therapy with HR of 0.44 (95\% CI: 0.23-0.86). The comparison between LAA occlusion and antiplatelet therapy showed no significant differences in the frequency of serious bleeding complications. These were comparable for LAA closure, placebo, antiplatelet therapy and NOACs.

The Health Authority in Ontario, Canada published a health technology assessment for patients with AF [12]. Five studies compared NOACS with warfarin and two studies compared LAA closure with warfarin. The evaluation showed that LAA closure for stroke reduction had similar results to NOACs with an OR of 0.85 . This was also true for mortality with an OR of 0.71. LAA closure was superior to NOACs in terms of cerebral bleeding (OR: 0.45).

Two of the meta-analyses focused on procedural success and complication. The analysis by Wei et al. included 38 studies with a total of 3585 patients [13]. The technical failure rate for LAA closure was $2 \%$, mean mortality was $3 \%$ and periprocedural stroke rate was $1 \%$. Thrombi on the device were found in $1 \%$ of patients and pericardial tamponade was found in $2 \%$ of patients.

The meta-analysis by Yerasi et al. included 49 studies with 12,415 patients [14]. A device for LAA closure was placed in $96.3 \%$ of patients. Total mortality during and after surgery was $0.28 \%$, stroke rate was $0.31 \%$ and severe bleeding rate was $1.71 \%$. Pericardial effusions were observed in $3.25 \%$ of patients.

Efficacy results should take into account that almost all data are from open observational studies and case series and are therefore subject to potential significant bias. However, the complication rates of LAA closure are comparable to those of the randomized studies.

\section{Prospective registries}

In the EWOLUTION study, 1020 patients with a WATCHMAN LAA implant procedure were prospectively followed for 2 years across 47 centers [15]. The indication for LAA occlusion was based on the ESC guidelines. The patients in the registry were relatively old (age $73.4 \pm 8.9$ years), and had a high risk for stroke. Of those included in the study, 311 patients had prior ischemic stroke or transient ischemic attack, 153 had prior intracerebral hemorrhage bleeding and 318 patients had a history of major bleeding. During the 2-year observation period 
Table 2. Ongoing studies in left atrial appendage occlusion.

\begin{tabular}{|c|c|c|c|c|c|}
\hline Study & Device & Control & Inclusion & Comparator & Primary end point \\
\hline STROKE CLOSE & 500 & 250 & History of intracerebral bleeding & Standard of care & $\begin{array}{l}\text { Stroke, systemic embolism and } \\
\text { life-threatening or severe bleeding as well } \\
\text { as all-cause mortality }\end{array}$ \\
\hline ASAP/TOO & 444 & 296 & Not suitable for oral anticoagulation & $\begin{array}{l}\text { Single antiplatelet or no } \\
\text { therapy }\end{array}$ & Stroke or systemic embolism \\
\hline CLOSURE AF & 756 & 756 & $\begin{array}{l}\text { HAS-BLED score } \geq 3 \text {, prior } \\
\text { intracranial/intraspinal bleed (BARC: } \\
\text { type } 3 c \text { ), hemorrhagic/bleeding } \\
\text { complication fulfilling BARC type 3a or } \\
\text { 3b: gastrointestinal tract, genitourinary } \\
\text { tract or respiratory tract bleeding, } \\
\text { where the patient is considered to be at } \\
\text { a persistently increased risk of bleeding }\end{array}$ & $\begin{array}{l}\text { Best medical treatment } \\
\text { (oral anticoagulation, } \\
\text { antiplatelet, no therapy) }\end{array}$ & $\begin{array}{l}\text { Stroke (including ischemic or hemorrhagic } \\
\text { stroke), systemic embolism, major bleeding } \\
\text { (BARC type 3-5) or cardiovascular or } \\
\text { unexplained death }\end{array}$ \\
\hline AMULET IDE & 534 & 534 & CHA2DS2-VASC score of $\geq 3$ & $\begin{array}{l}\text { Amplatzer - AMULET vs } \\
\text { WATCHMAN }\end{array}$ & $\begin{array}{l}\text { Procedure-related complications, all-cause } \\
\text { death, major bleeding through } 12 \text { months; } \\
\text { composite of ischemic stroke or systemic } \\
\text { embolism through } 18 \text { months; effective } \\
\text { device LAAO, defined as residual jet around } \\
\text { the device } \leq 5 \mathrm{~mm} \text { at the } 45 \text {-day visit }\end{array}$ \\
\hline
\end{tabular}

follow-up, 161 patients $(16.4 \%)$ died. There was a low rate of strokes $(\mathrm{n}=22 ; 1.3 / 100$ patient-years $)$ and major nonprocedural bleeding events ( $\mathrm{n}=47 ; 2.7 / 100$ patient-years). A device thrombus was observed in 34 patients $(4.1 \%)$ and was not correlated to drug regimen during follow-up $(\mathrm{p}=0.28)$.

The prospective Amulet Observational study recruited 1088 patients and initially followed them for 1 year $[16,17]$. The mean age was $75 \pm 9$ years; $65 \%$ of patients were male. The CHA2DS2-VASc and HAS-BLED scores were $4.2 \pm 1.6$ and $3.3 \pm 1.1$, respectively. The majority of patients $(83 \%)$ had contraindications to anticoagulation and $72 \%$ had a history of major bleeding. The observed annual ischemic stroke rate was $2.9 \%$. Device-related thrombus was observed in $1.7 \%$ of patients. In the first year, major bleeding occurred at a rate of $10.3 \%$. All-cause mortality was $8.4 \%$ at 1 year.

\section{Ongoing studies}

Currently, three studies on LAA closure in patients with AF are ongoing in which LAA closure is compared with a drug therapy or standard of care (Table 2).

The STROKECLOSE study, which is running in Scandinavia, is expected to enroll 750 patients (ClinicalTrials.gov Identifier: NCT02830152). Patients will be randomized in a 2:1 ratio to LAA closure or standard of care. Standard of care includes either the use of vitamin K antagonists, NOACs, antiplatelet therapy or no therapy. The primary inclusion criterion is the presence of intracerebral bleeding in CT or MRI scans within the last 6 months. The primary end point is the combination of stroke, SE and life-threatening or severe bleeding as well as all-cause mortality. After LAA closure, 6 months of therapy with aspirin with or without additional clopidogrel for 45 days is recommended.

The Assessment of the WATCHMAN ${ }^{\top M}$ Device in Patients Unsuitable for Oral Anticoagulation (ASAP/TOO) study is designed to evaluate 888 patients randomized 2:1 to LAA closure or drug therapy (ClinicalTrials.gov Identifier: NCT0292849). In the control group, patients will be treated with either a monotherapy with antiplatelet drugs or no therapy. Inclusion criterion is a CHA2DS2VASc score $\geq 2$ and the assessment of two study physicians that the patient with AF is not suitable for oral anticoagulation. The primary end point of the study is the time to stroke or SE over a period of 5 years. The primary safety end point is adverse events within the first 7 days after implantation or until discharge from hospital.

The Left Atrial Appendage CLOSURE in Patients with Atrial Fibrillation Compared with Medical Therapy (CLOSURE-AF) study has been designed to enroll 1512 patients receiving in 1:1 randomization LAA closure or best medical care (ClinicalTrials.gov Identifier: NCT03463317). Best medical care includes anticoagulation, including NOACs, antiplatelet therapy or no therapy. The study includes patients at high risk of bleeding under oral anticoagulation or contraindication to oral anticoagulation This includes at least one of the following conditions: HAS-BLED score $\geq 3$, prior intracranial/intraspinal bleed (BARC: type 3c), hemorrhagic/bleeding complication fulfilling BARC type $3 \mathrm{a}$ or $3 \mathrm{~b}$ : gastrointestinal tract, genitourinary tract or respiratory tract bleeding, where the 
patient is considered to be at a persistently increased risk of bleeding, in other words, the cause of bleeding cannot be successfully eliminated or chronic kidney disease (CKD) with eGFR $<30 \mathrm{ml} / \mathrm{min} / 1.73 \mathrm{~m}^{2}$. The primary end point of the study is survival time free of the composite of over 2 years of stroke (including ischemic or hemorrhagic stroke), SE, major bleeding (BARC type 3-5) or cardiovascular or unexplained death.

The Amulet IDE trial is an ongoing, prospective, randomized, multinational trial, designed to evaluate the safety and effectiveness of the AMPLATZER Amulet Left Atrial Appendage Occluder for stroke prevention in comparison to the WATCHMAN Left Atrial Appendage Closure Device in patients with nonvalvular AF [18]. The study recruits AF patients at high risk of stroke (CHA2DS2-VASc score of $\geq 3$ ) who are candidates for LAA occlusion. Patients are randomized in a 1:1 ratio between the AMPLATZER Amulet device (investigational) and the Boston Scientific WATCHMAN device (control). Each patient will be followed for 5 years. The planned sample size is 1088 patients. The primary end points are: a composite of procedure-related complications, or all-cause death, or major bleeding through 12 months (safety); a composite of ischemic stroke or SE through 18 months (effectiveness); and effective device LAAO, defined as residual jet around the device $\leq 5 \mathrm{~mm}$ at the 45 -day visit (mechanism of action).

The outcomes of these randomized studies will clarify which patient population with AF is particularly suitable for LAA closure.

\section{In which patients should LAA closure be considered?}

Most of the patients who are currently treated for LAA occlusion are patients with AF, who, in the opinion of the treating physician, are not eligible for long-term anticoagulation. The following patient populations fall into this category.

\section{Patients with intracranial hemorrhage}

Intracranial bleeding, especially intracerebral bleeding, is the most feared complication of oral anticoagulation in patients with AF. The mortality rate of intracerebral hemorrhage under anticoagulants is between 40 and 50\% [19]. Patients with a history of stroke and over 75 years of age are particularly at risk. A further risk factor is the combination of oral anticoagulation with antiplatelet drugs [19]. The risk of intracerebral hemorrhage is, however, significantly lower in the NOACs than with vitamin $\mathrm{K}$ antagonists. In patients in whom the source of bleeding can be identified and treated, oral anticoagulation with NOAC can restart after a period of 4-8 weeks [20,21]. A typical example would be an AF patient with poorly controlled hypertension who suffers a small basal ganglia bleed. If blood pressure is well controlled, anticoagulation can be resumed. Patients at high risk of rebleeding or where the source of bleeding could not be eliminated are candidates for LAA closure. These would include patients with intracerebral hemorrhage anticoagulated with warfarin and International Normalized Ratio (INR) in therapeutic range at the time of bleeding. Other examples are patients with severe small vessel disease and a high number of cortical microbleeds and patients with a positive family history and MRI imaging suggesting amyloid angiopathy. However, the bleeding risk of antithrombotic therapy recommended in the first 3 months after LAA implantation must be taken into account. That being said, in cases of extremely high bleeding risk, single antiplatelet treatment after LAA closure has also been used for some LAA occlusion systems [16,17].

\section{Patients with advanced renal failure}

Patients with renal failure have a particularly high risk of AF and conversely, the prevalence of severe renal dysfunction is increased in patients with AF. In this group of patients, oral anticoagulation is also difficult because NOACs are currently contraindicated in AF patients with a creatine clearance of $<30$ or $15 \mathrm{ml} / \mathrm{min}$. In patients with advanced renal failure, oral anticoagulation with warfarin is relatively ineffective and has a very high risk of bleeding [22]. In the meta-analysis of 13 publications from 11 cohorts including $>48,500$ patients with $>11,600$ warfarin users were analyzed [22]. In patients with $\mathrm{AF}$ and non-end-stage $\mathrm{CKD}$, warfarin resulted in a lower risk of ischemic stroke/thromboembolism (HR: 0.70; 95\% CI: 0.54-0.89; $\mathrm{p}=0.004$ ) and mortality (HR: 0.65; 95\% CI: 0.590.72; $\mathrm{p}<0.00001$ ), but had no effect on major bleeding (HR: 1.15; 95\% CI: 0.88-1.49; $\mathrm{p}=0.31$ ). In patients with AF and end-stage CKD, warfarin had no effect on the risks of stroke (HR: 1.12; 95\% CI: 0.69-1.82; $\mathrm{p}=0.65$ ) and mortality (HR: 0.96; 95\% CI: 0.81-1.13; $\mathrm{p}=0.60$ ), but increased the risks of major bleeding (HR: 1.30; 95\% CI: 1.08-1.56; $\mathrm{p}=0.005$ ) [2]. Patients with a creatine clearance of $<15 \mathrm{ml} / \mathrm{min}$ and dialysis patients would therefore be suitable candidates for LAA closure. However, no data from a randomized study are available in this patient population. 


\section{Patients with major gastrointestinal bleeding}

Another feared complication of oral anticoagulation is gastrointestinal bleeding. Gastrointestinal bleeds have a mortality rate of $2-3 \%$. With the exception of apixaban, the risk of these bleedings is higher in NOACS than in oral anticoagulation with warfarin [23]. In a Danish cohort study from 1996 to 2012, 4602 patients with a hospital admission due to gastrointestinal bleeding were analyzed [24]; 924 patients (27.1\%) did not resume antithrombotic treatment beyond 90 days after the initial event. Compared with nonresumption of treatment, a reduced risk of all-cause mortality was found in association with restart of oral anticoagulation (HR: 0.39; 95\% CI: 0.34-0.46) and a reduced risk of thromboembolism was found in association with restart of oral anticoagulation (HR: 0.41; 95\% CI: $0.31-0.54)$. Restarting oral anticoagulation alone was the only regime with an increased risk of major bleeding (HR: 1.37; 95\% CI: 1.06-1.77) compared with nonresumption of treatment. The difference in risk of recurrent gastrointestinal bleeding was not significant between patients who restarted an antithrombotic treatment regime and those who did not resume treatment [24].

If the cause of bleeding can be eliminated medically, endoscopically or surgically, patients can be anticoagulated again after gastrointestinal bleeding has occurred. If the source of bleeding cannot be eliminated and there is still a high risk of bleeding, LAA closure should be considered.

\section{Patients with a high risk of recurrence of ischemic stroke}

Oral anticoagulation is extremely effective in preventing strokes in patients with AF but cannot completely eliminate the risk. Patients who suffer a recurrent of ischemic stroke after optimal oral anticoagulation must first be examined to determine whether causes, other than AF, are responsible for the recurrence of stroke, such as large vessel disease. If this is not the case, a LAA closure may be considered in this population as well, if LAA thrombus formation is thought to be the cause of stroke.

\section{Elderly patients with high risk of bleeding \& falling}

The risk of serious bleeding complications increases with age and rises particularly in anticoagulated patients over 75 years of age [25]. This patient population is also at risk for falls, which can lead to either intracranial bleeding or fractures. In older patients with a high risk of bleeding and especially in patients with frequent falls, for example due to Parkinson's disease, epilepsy or another degenerative brain disease, LAA closure should also be discussed. However, it must be taken into account that the complication rate of the procedure is higher in older patients than in younger patients.

\section{Conclusion}

Patients with AF are frequently at an increased risk of stroke. NOAC treatment remains the primary therapeutic approach for stroke prevention in these patients. There are, however, patients who cannot tolerate long-term anticoagulation, largely because of an increased risk of bleeding, and in particular for these patients, catheter-based LAA closure has been developed as an alternative approach for stroke prevention in AF. Several randomized studies are currently ongoing to further determine the net clinical benefit of LAA closure in patients with AF at high ischemic risk and at high risk of bleeding, including the STROKECLOSE and the CLOSURE-AF study.

At present, LAA closure may in particular be considered for patients who cannot tolerate long-term anticoagulation or do have an absolute contraindication for anticoagulation. The antithrombotic treatment regime after LAA closure needs to be adapted to the bleeding risk of the patient.

The many technical aspects of LAA occlusion and the advantages and disadvantages of different devices are beyond the scope of this review. Details can be found in EHRA/EAPCI expert consensus statement [26].

\section{Future perspective}

Stroke is a main concern and complication in patients with AF. The results of several ongoing clinical studies will further determine which patients will benefit from catheter-based LAA closure, which has been developed as an alternative to anticoagulation for patients at high ischemic and high bleeding risk. Considering the poor compliance with oral anticoagulation more trials are needed to compare oral anticoagulation with NOACs with LAA occlusion over an extended period of time. 


\section{Executive summary}

Atrial fibrillation \& stroke

- Patients with atrial fibrillation (AF) have a fivefold increased risk of stroke compared with patients in sinus rhythm.

Prevention of stroke in AF with oral anticoagulation

- The risk of stroke in patients with AF can be reduced by $60-70 \%$ with vitamin $\mathrm{K}$ antagonists compared with placebo. Nonvitamin $\mathrm{K}$ oral anticoagulants are more effective than vitamin $\mathrm{K}$ antagonists, particularly in reducing the risk of intracerebral hemorrhage.

Risk groups for anticoagulation

- In some patients with AF, there is relative or absolute contraindication for oral anticoagulation. Treatment alternatives must be offered to these patients.

- One such treatment option is percutaneous interventional occlusion of the left atrial appendage (LAA).

Randomized studies for LAA occlusion

- There are two randomized trials comparing LAA closure with oral anticoagulation with warfarin in patients with AF.

- The PROTECT-AF and PREVAIL failed to show superiority of LAA closure compared with oral anticoagulation with warfarin in unselected patients with AF.

Meta-analyses

- Meta-analyses of the two randomized studies and data from open-label observational studies show no superiority of LAA closure over anticoagulation with nonvitamin $\mathrm{K}$ oral anticoagulants.

Prospective registries

- Two prospective registries for LAA occlusion with over 1000 patients each show a rate of ischemic stroke of $1.3-2.9 \%$ /year and severe bleeding events between 2.7 and $10 \%$.

Ongoing randomized studies

- Currently, three randomized trials are being conducted involving patients with AF and relative or absolute contraindication for long-term anticoagulation. In these studies, LAA closure is compared with standard of care.

- One randomized study compares the WATCHMAN and AMULET devices.

Suitable patients for the LAA closure

- Until the results of the randomized studies are available, potential candidates for LAA closure are patients with relative or absolute contraindication for long-term anticoagulation, such as patients after intracerebral or intracranial hemorrhage, high risk of recurrent ischemic stroke, severe renal dysfunction, severe gastrointestinal bleeding or advanced age in combination with frequent falls.

Financial \& competing interests disclosure

$\mathrm{H}-\mathrm{C}$ Diener received honoraria for participation in clinical trials, and contributed to advisory boards or oral presentations from: Abbott, BMS, Boehringer Ingelheim, Daiichi Sankyo, Medtronic, Novartis, Pfizer, Portola, Servier and WebMD. Global financial support for research projects was provided by Boehringer Ingelheim. Research grants were offered from the German Research Council (DFG), German Ministry of Education and Research (BMBF), European Union, NIH, Bertelsmann Foundation and Heinz Nixdorf Foundation. Within the past year H-C Diener served as editor of Arzneimitteltherapie, Info Neurologie \& Psychiatrie and Neurologie up2date, as co-editor of Cephalalgia and on the editorial board of Lancet Neurology. H-C Diener chairs the Treatment Guidelines Committee of the German Society of Neurology and contributed to the EHRA and ESC guidelines for the treatment of atrial fibrillation. U Landmesser received speaker and consulting honoraria from Abbott, Boston Scientific, Biotronik, Amgen, Sanofi, Novartis, the Medicines Company, Bayer and Boehringer Ingelheim. The authors have no other relevant affiliations or financial involvement with any organization or entity with a financial interest in or financial conflict with the subject matter or materials discussed in the manuscript apart from those disclosed.

No writing assistance was utilized in the production of this manuscript.

\section{Open access}

This work is licensed under the Attribution-NonCommercial-NoDerivatives 4.0 Un-ported License. To view a copy of this license, visit http://creativecommons.org/licenses/by-nc-nd/4.0/ 


\section{References}

Papers of special note have been highlighted as: $\bullet$ of interest; $\bullet \bullet$ of considerable interest

1. Kirchhof P, Benussi S, Kotecha D et al. 2016 ESC guidelines for the management of atrial fibrillation developed in collaboration with EACTS. Eur. Heart J. 37(38), 2893-2962 (2016).

2. Ruff CT, Giugliano RP, Braunwald E et al. Comparison of the efficacy and safety of new oral anticoagulants with warfarin in patients with atrial fibrillation: a meta-analysis of randomized trials. Lancet 383(9921), 955-962 (2014).

3. Hart R, Benavente O, McBride R, Pearce L. Antithrombotic therapy to prevent stroke in patients with atrial fibrillation: a meta-analysis. Ann. Intern. Med. 131(7), 492-501 (1999).

4. Lakkireddy D, Windecker S, Thaler D et al. Rationale and design for AMPLATZER Amulet Left Atrial Appendage Occluder IDE randomized controlled trial (Amulet IDE trial). Am. Heart J. 211, 45-53 (2018).

5. Reddy VY, Sievert H, Halperin J et al. Percutaneous left atrial appendage closure vs warfarin for atrial fibrillation: a randomized clinical trial. JAMA 312(19), 1988-1998 (2014).

- Randomized trial comparing left atrial appendage (LAA) occlusion with antithrombotic therapy in patients with atrial fibrillation.

6. Holmes DR Jr, Kar S, Price MJ et al. Prospective randomized evaluation of the WATCHMAN left atrial appendage closure device in patients with atrial fibrillation versus long-term warfarin therapy: the PREVAIL trial. J. Am. Coll. Cardiol. 64(1), 1-12 (2014).

- Randomized trial comparing LAA occlusion with antithrombotic therapy in patients with atrial fibrillation.

7. Osmancik P, Tousek P, Herman D et al. Interventional left atrial appendage closure vs novel anticoagulation agents in patients with atrial fibrillation indicated for long-term anticoagulation (PRAGUE-17 study). Am. Heart J. 183, 108-114 (2017).

- First randomized trial to compare LAA occlusion with nonvitamin $\mathrm{K}$ oral anticoagulants.

8. Reddy VY, Doshi SK, Kar S et al. 5-Year outcomes after left atrial appendage closure: from the PREVAIL and PROTECT AF trials. J. Am. Coll. Cardiol. 70(24), 2964-2975 (2017).

9. Bode WD, Patel N, Gehi AK. Left atrial appendage occlusion for prevention of stroke in nonvalvular atrial fibrillation: a meta-analysis. $J$. Interv. Card. Electrophysiol. 43(1), 79-89 (2015).

10. Li X, Wen SN, Li SN et al. Over 1-year efficacy and safety of left atrial appendage occlusion versus novel oral anticoagulants for stroke prevention in atrial fibrillation: a systematic review and meta-analysis of randomized controlled trials and observational studies. Heart Rhythm 13(6), 1203-1214 (2016).

11. Sahay S, Nombela-Franco L, Rodes-Cabau J et al. Efficacy and safety of left atrial appendage closure versus medical treatment in atrial fibrillation: a network meta-analysis from randomized trials. Heart 103(2), 139-147 (2017).

12. Health Quality Ontario. Left atrial appendage closure device with delivery system: a health technology assessment. Ont. Health Technol. Assess. Ser. 17(9), 1-106 (2017).

13. Wei $\mathrm{Z}$, Zhang $\mathrm{X}$, Wu H et al. A meta-analysis for efficacy and safety evaluation of transcatheter left atrial appendage occlusion in patients with nonvalvular atrial fibrillation. Medicine (Baltimore) 95(31), e4382 (2016).

14. Yerasi C, Lazkani M, Kolluru P et al. An updated systematic review and meta-analysis of early outcomes after left atrial appendage occlusion. J. Interv. Cardiol. 31(2), 197-206 (2018).

15. Boersma LV, Ince H, Kische $S$ et al. Evaluating real-world clinical outcomes in atrial fibrillation patients receiving the WATCHMAN left atrial appendage closure technology. Circ. Arrhythm. Electrophysiol. 12(4), e006841 (2019).

16. Landmesser U, Tondo C, Camm J et al. Left atrial appendage occlusion with the AMPLATZER Amulet device: one-year follow-up from the prospective global Amulet observational registry. EuroIntervention 14(5), e590-e597 (2018).

17. Landmesser U, Schmidt B, Nielsen-Kudsk JE et al. Left atrial appendage occlusion with the AMPLATZER Amulet device: periprocedural and early clinical/echocardiographic data from a global prospective observational study. EuroIntervention 13(7), 867-876 (2017).

18. Lakkireddy D, Windecker S, Thaler D et al. Rationale and design for AMPLATZER Amulet Left Atrial Appendage Occluder IDE randomized controlled trial (Amulet IDE trial). Am. Heart J. 211, 45-53 (2019).

19. Hart RG, Diener H-C, Yang S et al. Intracranial hemorrhage in atrial fibrillation patients during anticoagulation with warfarin or dabigatran: the RE-LY trial. Stroke 43(6), 1511-1517 (2012).

20. Diener H-C, Aisenberg J, Ansell J et al. Choosing a particular oral anticoagulant and dose for stroke prevention in individual patients with nonvalvular atrial fibrillation: part 2. Eur. Heart J. 38(12), 860-868 (2017).

21. Diener H-C, Aisenberg J, Ansell J et al. Choosing a particular oral anticoagulant and dose for stroke prevention in individual patients with nonvalvular atrial fibrillation: part 1. Eur. Heart J. 38(12), 852-859 (2017).

22. Dahal K, Kunwar S, Rijal J, Schulman P, Lee J. Stroke, major bleeding and mortality outcomes in warfarin users with atrial fibrillation and chronic kidney disease: a meta-analysis of observational studies. Chest 149(4), 951-959 (2016).

23. Lopez-Lopez JA, Sterne JAC, Thom HHZ et al. Oral anticoagulants for prevention of stroke in atrial fibrillation: systematic review, network meta-analysis and cost-effectiveness analysis. BMJ 359, j5058 (2017). 
24. Staerk L, Lip GY, Olesen JB et al. Stroke and recurrent hemorrhage associated with antithrombotic treatment after gastrointestinal bleeding in patients with atrial fibrillation: nationwide cohort study. BMJ 351, h5876 (2015).

25. Kim IS, Kim HJ, Kim TH et al. Nonvitamin K antagonist oral anticoagulants have better efficacy and equivalent safety compared to warfarin in elderly patients with atrial fibrillation: a systematic review and meta-analysis. J. Cardiol. 72(2), 105-112 (2018).

26. Glikson M, Wolff R, Hindricks G et al. EHRA/EAPCI expert consensus statement on catheter-based left atrial appendage occlusion: an update. EuroIntervention 15(13), 1133-1180 (2020).

•• European consensus statement on interventional LAA occlusion. 